\title{
Research and Implementation of the Satellite Channel Two-State Model Under the Cloudy and Foggy Weather
}

\author{
Yecai GUO ${ }^{1,2, a}$, Runzhi ZHOU ${ }^{1}$, Weijuan ZHAO ${ }^{1}$, Jiahui WU ${ }^{1}$, Ningxiao GU $^{1}$ \\ ${ }^{1}$ School of Electronic \& Information Engineering, Nanjing University of Information Science and \\ Technology, Nanjing, 210044, China \\ ${ }^{2}$ Jiangsu Collaborative Innovation Center on Atmospheric Environment and Equipment, \\ Nanjing, 210044, China \\ aemail: guo-yecai@163.com
}

Keywords: Cloudy and Foggy, Propagation Characteristics, Channel Model, Markov Model.

\begin{abstract}
In order to research the propagation characteristics of the satellite channel under the environment of the cloudy and foggy, a satellite channel two-state Markov model under the cloudy and foggy weather is proposed. Firstly, the influences of the multipath effect, shadowing effects, and cloudy and foggy weather on the satellite channel are analyzed. The corresponding Suzuki model and Nakagami model are established based on the channel propagation characteristics under the cloudy and foggy conditions. Then the two-state Markov model is introduced into satellite channel model to achieve the transformation of the channel characteristics with the change of cloudy and foggy weather. Finally, the simulation method of the satellite channel two-state Markov model is designed. The results show that the simulation results of the proposed model are quite consistent with those of the theoretical analyses.
\end{abstract}

\section{Introduction}

With the continuous development of human society, the demand for communication is increasing and the requirement for the communication quality is becoming higher. In the satellite communication system, the wireless communication environment will affect the quality of the communication system directly. Therefore, it is very significant to establish the mathematical model based on the channel propagation characteristics. At present, the modeling of satellite channel mainly concentrates on the ground environment with various obstacles[1][2]. In [3][4], the mobile satellite channel characteristics under the different ground environments, such as trees, buildings, were researched. For the high frequency satellite communication system, the researches of [5][6] indicated that cloudy, rainfall, and other atmospheric factors also impact on the satellite communication, so the influences of atmosphere environment on channel propagation characteristics can't be neglected.

In order to research the influences of clouds and fog on satellite communications, in this paper, the channel propagation characteristics are first analyzed. Then the Nakagami model and Suzuki model are established based on the channel propagation characteristics under the cloudy and foggy conditions. Finally, the satellite channel two-state Markov model is established and simulated.

\section{Satellite Channel Propagation Characteristics Under Cloudy and Foggy Conditions}

At present, in the classic satellite channel modeling, the influence of the ground environment is considered,whereas the atmospheric attenuation is ignored usually. To research the influence of clouds and fog attenuation, we assume that the ground environment is ideal. For the satellite-ground station link, the effects of the ground environment can't be considered, whereas the propagation characteristics of the satellite channel under the environment of the cloudy and foggy are discussed.

Multipath effect. Multipath effect existing widely in each frequency band of satellite communication is caused by the reflecting, scattering, and diffraction. The received signals consist of the waveforms from different path. Rayleigh distribution, Rice distribution, and Nakagami 
distribution can be used to describe the multipath effect. Nakagami distribution is a kind of distribution with parameter $m$ [7]. With different value of $m$, Nakagami distribution can describe the different fading of the channel environment. It has better flexibility and adaptability than Rayleigh and Rice distribution. The probability density function of the Nakagami distribution can be expressed as

$$
f_{N}(r)=\frac{2 m^{m} r^{2 m-1}}{\Omega^{m} \Gamma(m)} \exp \left(-\frac{m r^{2}}{\Omega}\right) \quad r \geq 0
$$

where, $\Omega=E\left[r^{2}\right]$ is the average power of the received signal. $\Gamma(\cdot)$ is gamma function. $m=\Omega^{2} / E\left\{\left[r^{2}-\Omega^{2}\right]^{2}\right\}$ is the shape factor of the Nakagami distribution, which describes the fading degree of the propagation field caused by the interference of the multipath phenomena. When the value of $m$ becomes smaller, the attenuation of the signal becomes more serious. The Nakagami probability density function with different values of parameter $m$ is shown in Fig.1.

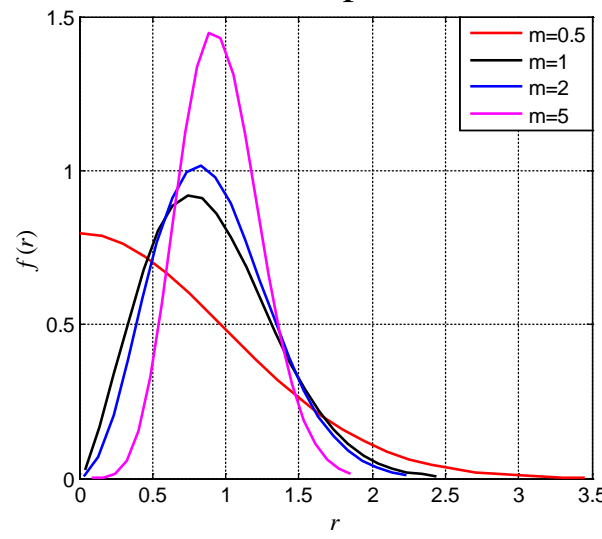

Fig.1. The Nakagami PDF for various values of $m$

Showing effect. When the satellite signals pass through the trees, buildings, and clouds, the shadowing effect is caused by the blocking, reflection, and refraction of these obstacles. The received signals will fade. Many studies show that the shadowing effect can be described by Lognormal distribution[8], its probability density function can be expressed as

$$
f_{L N}(r)=\frac{20}{\sqrt{2 \pi} \sigma_{u}^{2} r \ln (10)} \exp \left(-\frac{\left(20 \log _{10} r-m_{u}\right)^{2}}{2 \sigma_{u}{ }^{2}}\right) \quad r \geq 0
$$

where, $\sigma_{u}, m_{u}$ are the standard deviation and mean of the direct component.

Clouds and fog attenuation. Clouds and fog belong to the water suspension body, their diameters are generally less than $0.01 \mathrm{~cm}$. For the high frequency band of satellite communications, the attenuation caused by the water suspension body will be serious. With the increase of frequency and the decrease of the satellite elevation angle, the clouds and fog attenuation will increase. The ITU-R provides methods to predict the attenuation due to clouds and fog on Earth-space paths[9]. The attenuation within a cloud or fog can be written as

$$
A_{c}=\frac{L K_{1}}{\sin \theta}
$$

where $\theta\left(10^{\circ} \leq \theta \leq 90^{\circ}\right)$ is the elevation angle. $L$ is the columnar water content. $K_{1}$ is attenuation coefficient, it is relevant to the frequency and the dielectric constant of the water.

\section{Satellite Channel Markov Model Under Cloudy and Foggy Conditions}

In different weather, the signals will appear different attenuation on the channel transmission. In this paper, the channel propagation characteristics under the cloudy and foggy conditions are considered. The corresponding models are also established.

The Suzuki model under cloudy weather condition. In the cloudy weather, there are greatly thick clouds and large amount clouds. The received signals are affected by multipath effect and 
shadowing fading caused by clouds. In this case, the channel characteristics can be described by Suzuki model. Suzuki model is generated by Rayleigh distribution and Lognormal distribution. The probability density function of the received signal envelope $r$ can be expressed as

$$
f_{s}(r)=\frac{20 r}{\sigma^{2} \sigma_{u} \sqrt{2 \pi} \ln (10)} \int_{0}^{\infty} \frac{1}{z^{3}} \exp \left(-\frac{(r / z)^{2}}{2 \sigma^{2}}-\frac{\left(20 \log _{10} z-m_{u}\right)^{2}}{2 \sigma_{u}{ }^{2}}\right) d z \quad r \geq 0
$$

where, $\sigma^{2}$ is the average power of multipath component. $\sigma_{u}$ and $m_{u}$ are the standard deviation and mean of $\ln (z)$. In the cloudy weather, the cumulative distribution function of the received signal envelope $r$ is given by

$$
F_{S}(r)=\int_{0}^{r} f_{S}(x) \mathrm{d} x=\frac{20}{\sigma^{2} \sigma_{u} \sqrt{2 \pi} \ln (10)} \int_{0}^{r} \int_{0}^{\infty} \frac{x}{z^{3}} \exp \left(-\frac{(x / z)^{2}}{2 \sigma^{2}}-\frac{\left(20 \log _{10} z-m_{u}\right)^{2}}{2 \sigma_{u}{ }^{2}}\right) \mathrm{d} z \mathrm{~d} x
$$

The Nakagami model of fog weather condition. In the foggy weather, the signal suffers from fog attenuation through the channel transmission. The received signal is the superposition of multiple paths. Meanwhile, the transmission signals have different attenuation within different concentrations of the fog. The different signal attenuation caused by the fog can be described by Nakagami model. The probability density function of the Nakagami model can be expressed as (1). Nakagami model is used to describe the light fog attenuation with $m=2$, and the thick fog attenuation with $m=1$. In the foggy weather, the cumulative distribution function of the received signal envelope $r$ is given by

$$
F_{N}(\rho)=\frac{\Gamma\left(m, m \rho^{2}\right)}{\Gamma(m)}
$$

where, $\rho=r / \sqrt{\Omega}$. $\Gamma(\cdot)$ is gamma function; $\Gamma(a, b)=\int_{0}^{b} x^{a-1} e^{-x} \mathrm{~d} x$.

The satellite channel two-state Markov model. With the change of cloudy and foggy, Nakagami model and Suzuki model be used to describe the fading characteristics and these two methods will dynamically and randomly switch each other. Markov chain model is used here to achieve this stochastic switching process and accurately describe the dynamic characteristics of satellite channel. Markov model can be described by the state probability matrix $\boldsymbol{S}$ and state transition matrix $\boldsymbol{P}$. The state probability matrix $\boldsymbol{S}=\left[s_{c} s_{f}\right]$, which is a collection of the probability that each state might occur. The state transition matrix $\boldsymbol{P}$ is formed by the state transition probability. It can be written as $\boldsymbol{P}=\left[p_{c c}, p_{c f} ; p_{f c}, p_{f f}\right]$. Where, $p_{c c}$ and $p_{f f}$ express the probability of the channel maintaining in cloudy and foggy weather, respectively, while $p_{c f}$ and $p_{f c}$ represent the probability of the channel from the cloudy to the foggy weather or from the foggy to the cloudy weather. If the initial state is known, the next state can be obtained by a random variable of uniform distribution compared with the current state of transition probability. So, the long-term forecast of the channel state can be realized under the cloudy and foggy weather. The two-state Markov transition model is depicted in Fig.2.

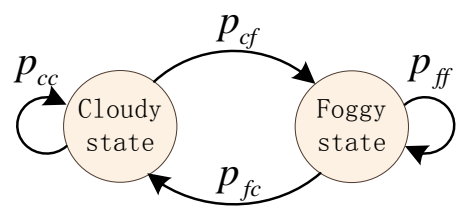

Fig. 2. Two-state Markov transition model

The probability density function of the total received signal envelope can be stated as $f(r)=s_{c} f_{s}(r)+s_{f} f_{N}(r)$

\section{The Implementation Method of the Satellite Channel Model}

The channel model can describe the channel propagation characteristics by mathematical method, but it needs to be realized with hardware or software in research and production. Therefore, it is very necessary to research the realization of the satellite channel theoretical model. 
The realization of colored Gaussian process. All the channel models are based on a number of uncorrelated colored Gaussian processes. So, the implementation method of colored Gaussian random processes is discussed firstly. In this paper, the method of sun-of-sinusoids (SOS) is employed for designing colored Gaussian random processes[10]. The main idea of the SOS is to approximate colored Gaussian processes by a finite sum of sinusoidal waveforms, and shown in Fig.3. The deterministic Gaussian process is given by $\mu_{i}(t)=\sum_{n=1}^{N_{i}} c_{i, n} \cos \left(2 \pi f_{i, n} t+\theta_{i, n}\right)$, where $N_{i}$ denotes the number of sinusoids. The quantities $c_{i, n}, f_{i, n}$, and $\theta_{i, n}$ are the simulation model parameters called as the Doppler gains, the Doppler frequencies, and the Doppler phases, respectively. Doppler phases $\theta_{i, n}$ are generated from the uniform random number of $(0,2 \pi]$. Doppler gains $c_{i, n}$ is defined as $c_{i, n}=\sigma \sqrt{\frac{2}{N_{i}}}, i=1,2, \ldots 5$. Doppler frequencies $f_{i, n}$ is defined as $f_{i, n}=f_{\max } \sin \left[\frac{\pi}{2 N_{i}}\left(n-\frac{1}{2}\right)\right], i=1,2,4,5 \quad$ or $\quad f_{i, n}=\frac{f_{c}}{\sqrt{\ln 2}} \operatorname{erfinv}\left(\frac{2 n-1}{2 N_{i}}\right), i=3$. Where $\sigma$ denotes the standard deviation, $f_{\max }$ is the maximum Doppler frequency, $f_{c}$ is the cut-off frequency of $3 \mathrm{~dB}$, and $\operatorname{erfinv}(\cdot)$ is the inverse error function.

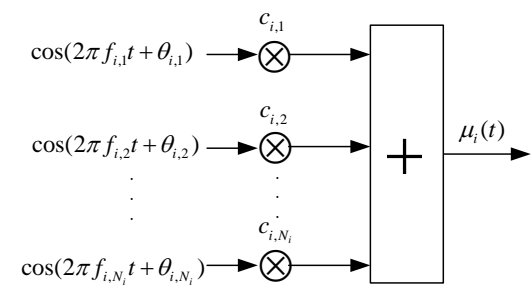

Fig.3. The diagram of sun-of-sinusoids

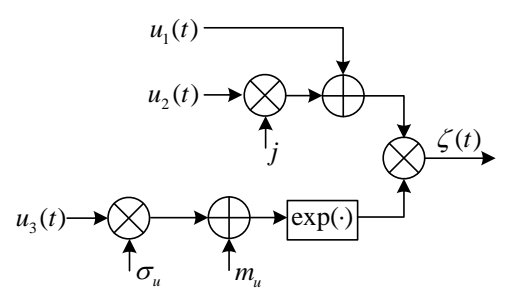

Fig.4. Suzuki model

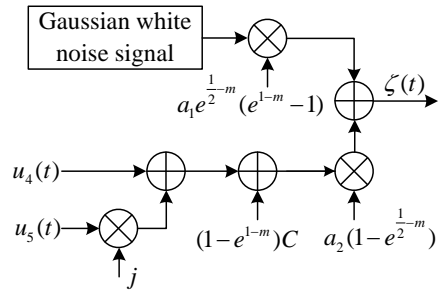

Fig.5. Nakagami model

The Suzuki channel simulation model. Suzuki channel model is used to describe the channel influence of multipath shadowing fading, and regarded as the product of a Rayleigh process and a Lognormal process. The Rayleigh process and lognormal process are made up of the uncorrelated colored Gaussian processes $\mu_{i}(t)$. Its implementation block diagram is given in Fig.4.

The Nakagami channel simulation model. When the value of $m$ is different, the Nakagami distribution can conform to the Gaussian distribution, Rayleigh distribution, and the Rice distribution. So we can achieve the Nakagami channel model by these distributions. In this paper, the Nakagami channel model is generated with Gaussian distribution and Rayleigh distribution. The received signal of the Nakagami channel can be expressed as

$$
r_{\text {Nak }}(t)=a_{1} e^{\frac{1}{2}-m}\left(e^{1-m}-1\right) g(t)+a_{2}\left(1-e^{\frac{1}{2}-m}\right)\left[\left(1-e^{1-m}\right) C+r_{\text {ray }}(t)\right]
$$

where $a_{1}=1 /(\sqrt{e}-1)$ and $a_{2}=\sqrt{e}(\sqrt{e}-1), g(t)$ is Gaussian white noise signal, and $r_{\text {ray }}(t)$ is the complex envelope of the Rayleigh fading signal generated by the uncorrelated real Gaussian random processes $\mu_{1}(t)$ and $\mu_{1}(t), C$ is correction factor. The implementation method is shown in Fig.5.

\section{Simulation Analysis}

In order to validate the satellite channel two-state Markov model, the relevant data are used to detect the performance of the proposed channel model. In the two-state satellite channel Markov simulation, the parameters are set as follow: the variance of Rayleigh was 0.08 , the standard deviation of Lognormal was $1 \mathrm{~dB}$ and the mean value was $-8 \mathrm{~dB}$, Nakagami model is used to describe the light fog attenuation with $m=2$, and the thick fog attenuation with $m=1$. The envelope of the received signal is shown in Fig.6. 


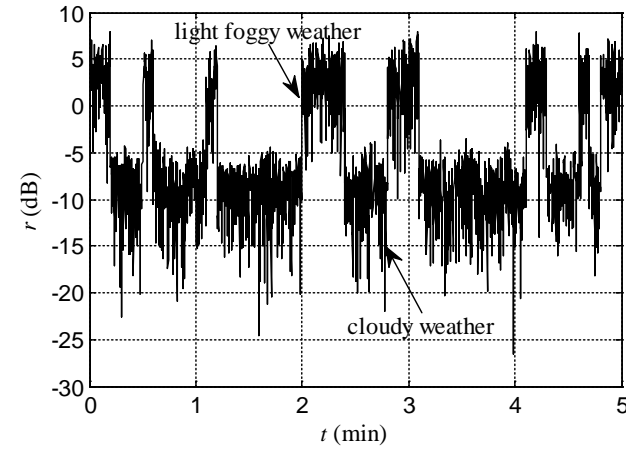

(a) Light foggy-cloudy

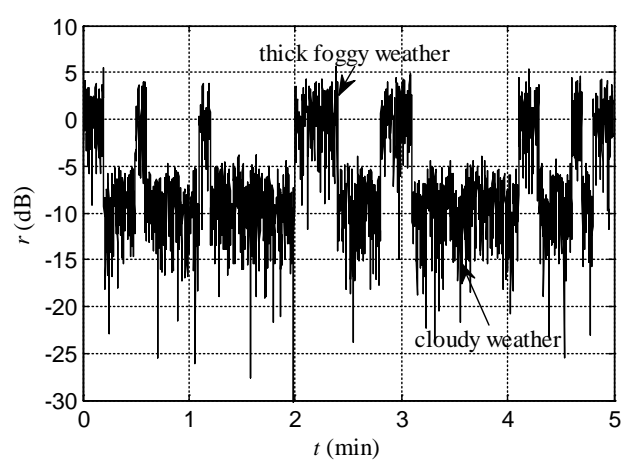

(b) Thick foggy-cloudy

Fig.6. The received signal envelope

Fig.6 shows that the transmission signals have different degree of fading caused by the clouds and fog. With the change of weather, the two channel models can happen to transform. In the different concentrations of fog, the signal attenuation is different, too.

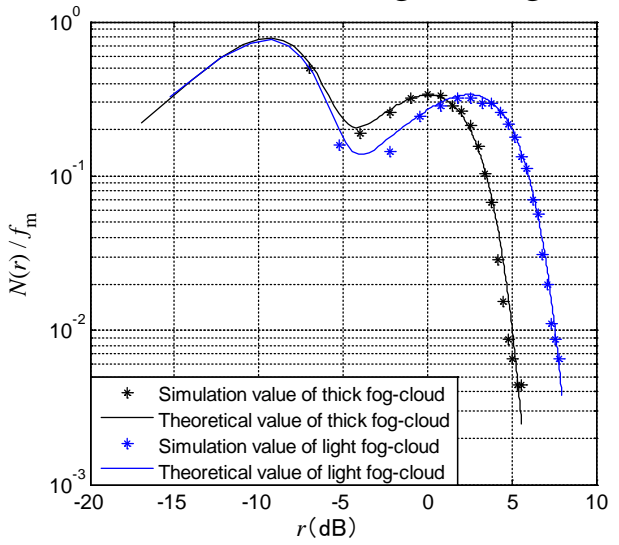

Fig. 7 The normalized level crossing rate

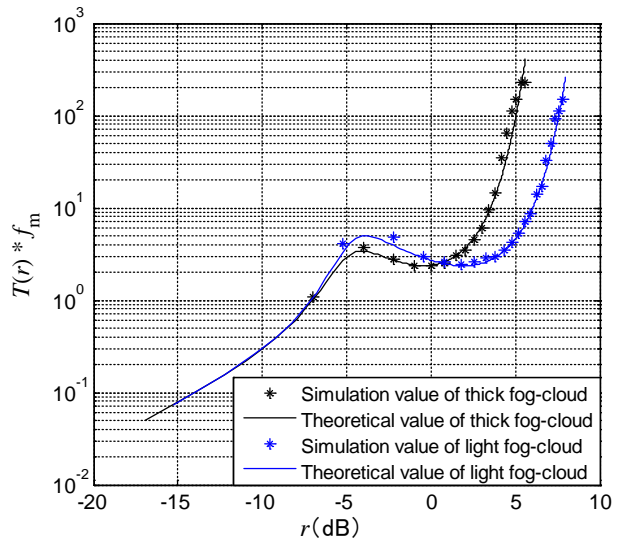

Fig. 8 The normalized average duration of fade

The level crossing rate(LCR) and the average duration of fade(ADF) functions are important second order statistical quantities and used for reflecting the characteristics of the fading channel. Fig.7 and Fig. 8 show the normalized level crossing rate and normalized average duration of fade of the two-state Markov satellite channel. From Fig.7 and Fig.8, we may conclude that the statistical characteristics curves of the simulation are sufficiently close to those of the theoretical model. Simulations demonstrate that the proposed channel two-state Markov model can be used to describe the propagation characteristics of satellite channel under the cloudy and foggy weather.

\section{Conclusion}

In this paper, the satellite channel two-state Markov model is established under the cloudy and foggy weather and its implement method is researched. The two-state Markov model consists of Suzuki model and Nakagami model based on the propagation characteristics of the wireless channel. The two-state Markov model effectively overcomes the problem that the single state model can not reflect the influences of satellite channel due to the weather change. The simulation results show that the satellite channel two-state Markov model can be used to describe the actual satellite channel propagation characteristics. It provides certain basis to research the effect of atmospheric attenuation on the satellite communication.

\section{Acknowledgements}

Project supported the Major Project of Nature Science Foundation of Higher Education Institution of Jiangsu Province, China(Grant No.13KJA510001), Jiangsu Scientific Research Achievements in Industrialization Project, China(JHB 2012-9), and Jiangsu Provincial College 
Students' innovative training program(201310300051Y)

\section{References}

[1] Lutz E. Modeling of the land mobile satellite communications channel[C]. IEEE-APS Topical Conference on Antennas and Propagation in Wireless Communications(APWC), Torino, 2013: 199-202.

[2] Fontan F P. Channel Modeling for Land Mobile Satellite Services[C]. Proceedings of the Fourth European Conference on Antennas and Propagation(EUCAP), Barcelona, 2010:1-5

[3] Kourogiorgas C I, Kvicera M, Skraparlis D, et al. Modeling of First-Order Statistics of LMS Channel under Tree Shadowing for Various Elevation Angles at L-Band[C]. $8^{\text {th }}$ European Conference on Antennas and Propagation(EuCAP), The Hague, 2014: 2273-2277.

[4] Seyedi Y, Shirazi M, Moharrer A, et al. Use of shadowing moments to statistically model mobile satellite channels in urban environments[J]. IEEE Transactions on Wireless Communications, 2013,12(8): 3760-3769.

[5] Yang Ruike, Li Li, Zhao Zhenwei, Lu Tongya. Cloud Simulation and Attenuation at Ka Band on Slant Path[C]. Cross Strait Quad-Regional Radio Science and Wireless Technology Conference(CSQRWC), Chengdu, 2013:300-303.

[6] Zhang Xiuzai, Guo Yecai, Yang Changjun. The Meteorological Satellite Composite Channel Model under Cloudy Weather Conditions[J].Journal of Computational Information Systems, 2013, 9(13): 5337-5344.

[7] Shen Dongya, Cui Yanni, Zhang Aofeng, et al. A simple simulation method for Nakagami fading channel[C]. International Conference on Microwave and Millimeter Wave Technology(ICMMT), Chengdu, 2010:160-163.

[8] Rafiq G, Patzold M. The impact of shadowing on the capacity of mobile fading channels[C].4th International Symposium on Wireless Communication Systems, Trondheim, 2007: 209-214.

[9] Louis J. Ippolito Jr. Satellite communications systems engineering[M]. John Wiley\& Sons Inc, Publication.2008.

[10] Patzoid M, Cheng-Xiang Wang, Hogstad B O. Two new Sum-of-Sinusoids-Based methods for the efficient generation of multiple uncorrelated Rayleigh fading waveforms[J]. IEEE Transactions on Wireless Communications. 2009, 8(6):3122-3131. 